\title{
Phylogenetic analysis of the vertebrate Excitatory/Neutral Amino Acid Transporter (SLC1/EAAT) family reveals lineage specific subfamilies
}

\author{
Matthias Gesemann*1, Annegret Lesslauer 1,2, Colette M Maurer' ${ }^{1}$, Helia B Schönthaler1,3 and Stephan CF Neuhaus**1
}

\begin{abstract}
Background: The composition and expression of vertebrate gene families is shaped by species specific gene loss in combination with a number of gene and genome duplication events (R1, R2 in all vertebrates, R3 in teleosts) and depends on the ecological and evolutionary context. In this study we analyzed the evolutionary history of the solute carrier 1 (SLC1) gene family. These genes are supposed to be under strong selective pressure (purifying selection) due to their important role in the timely removal of glutamate at the synapse.

Results: In a genomic survey where we manually annotated and analyzing sequences from more than 300 SLC1 genes (from more than 40 vertebrate species), we found evidence for an interesting evolutionary history of this gene family. While human and mouse genomes contain 7 SLC1 genes, in prototheria, sauropsida, and amphibia genomes up to 9 and in actinopterygii up to 13 SLC1 genes are present. While some of the additional s/c1 genes in ray-finned fishes originated from R3, the increased number of SLC1 genes in prototheria, sauropsida, and amphibia genomes originates from specific genes retained in these lineages.

Phylogenetic comparison and microsynteny analyses of the SLC1 genes indicate, that theria genomes evidently lost several SLC1 genes still present in the other lineage. The genes lost in theria group into two new subfamilies of the s/c1 gene family which we named s/c1a8/eaat6 and slc1a9/eaat7.

Conclusions: The phylogeny of the SLC1/EAAT gene family demonstrates how multiple genome reorganization and duplication events can influence the number of active genes. Inactivation and preservation of specific SLC1 genes led to the complete loss of two subfamilies in extant theria, while other vertebrates have retained at least one member of two newly identified SLC1 subfamilies.
\end{abstract}

\section{Background}

Genomes of extant species are shaped by extensive gene loss and duplication events. In the radiation of vertebrate species, two whole genome duplication events at the base of the vertebrate lineage (approximately 500 million years (Mya) ago) [1-3] and a third round of genome duplication at the base of the teleost lineage (about 350 Mya ago [47]) are proposed to play a crucial role [1-3]. Duplicated genes are expected to be functionally redundant and

*Correspondence: matthias.gesemann@imls.uzh.ch, stephan.neuhauss@imls.uzh.ch University of Zurich, Institute of Molecular Life Sciences, Winterthurerstrasse 190, CH-8057 Zurich, Switzerland

Full list of author information is available at the end of the article therefore released of selective pressure, consequently leading to gene loss. Nevertheless many duplicated paralogs are retained in modern genomes, for instance an estimated $15-24 \%$ of duplicated paralogs are present in extant teleost genomes [6,8-10]. The duplication-complementation-degeneration model seeks to explain the retention of duplicated genes by subfunctionalization, where the function of the essential ancestral gene is distributed to two genes, each fulfilling part of the ancestral gene's function due to regulatory mutations $[11,12]$. Another most interesting possible event following gene duplication is neofunctionalization, in which one of the two paralogous genes is free to acquire a new function 
that differs from the ancestral gene, due to the essential (ancestral) function being carried out by the unchanged paralog. Such functionalization events may pave the way for speciation. An alternative driving force in speciation might be divergent resolution, where random paralog losses in two allopatric populations can lead to diversity [13-15]. While whole genome duplications are one way to increase the gene repertoire within a genome, tandem duplication events as well as lineage specific gene loss are other means to change the number of active genes (e.g. $[16,17]$.

In the context of lineage specific gene loss and duplication events, we studied members of the vertebrate $s l c 1$ gene family of neutral and excitatory amino acid transporters (EAATs), whose presence on glia cells or neurons is indispensable for precise and sustained synaptic activity [18-22]. EAATs are involved in the removal of glutamate from excitatory synapses, a process not only essential to ensure precise termination of synaptic transmission but also to avoid neurotoxic accumulation implicated in a number of diseases [23]. Moreover, transport of glutamate is associated with an increased chloride conductance across the membrane, which hyperpolarizes cells [24]. Based on these important functions high-affinity glutamate transporters in the nervous system are supposed to be under strong purifying selection (pressure to stay the same).

In mammals, five EAAT genes (EAAT 1-5) together with two neutral amino acid transporters form the 'solute carriers 1' (SLC1) gene family (for detailed information on nomenclature, refer to Table 1). In these species EAAT genes have diversified both in spatial and temporal expression and in functional properties and are expressed

Table 1: Gene Aliases in Different Nomenclatures.

\begin{tabular}{lll}
\hline $\begin{array}{l}\text { SLC1 } \\
\text { nomenclature }\end{array}$ & $\begin{array}{l}\text { EAAT } \\
\text { nomenclature }\end{array}$ & $\begin{array}{l}\text { Other } \\
\text { aliases }\end{array}$ \\
\hline SLC1A1 & EAAT3 & EAAC1 \\
SLC1A2 & EAAT2 & GLT-1 \\
SLC1A3 & EAAT1 & GLAST, \\
SLC1A4 & GLAST-1 \\
SLC1A5 & & \\
SLC1A6 & & \\
SLC1A7 & EAAT4 & \\
\hline
\end{tabular}

SLC1 = Solute Carrier Family 1; EAAT = Excitatory Amino Acid Transporter; both nomenclatures originally describe the gene families in man. SLC1A4 and SLC1A5 belong phylogenetically to the SLC1 family, mediate however different functions than EAAT and are therefore missing from the EAAT nomenclature. Data are based on the official HUGO instructions http:// www.genenames.org. at glutamatergic synapses throughout the central nervous system. SLC1A3/EAAT1 and SLC1A2/EAAT2 are predominantly expressed in glia cells and presumably mediate the main load of glutamate re-uptake while eliciting only small chloride currents $[18,20]$. The neuronal transporters, SLC1A1/EAAT3, SLC1A6/EAAT4 and SLC1A7/ EAAT5, exhibit larger chloride currents, especially the cerebellar SLC1A6/EAAT4 and the retina-specific SLC1A7/EAAT5 $[18,20]$.

In teleosts, given the evidence for a third round of genome duplication (R3), an increased number of SLC1 genes are expected in their genomes. We indeed identified up to 13 SLC1s in fish genomes. While some of these additional SLC1 genes clearly are the consequence of the teleost specific R3, others are evidently members of two additional subfamilies (now called SLC1A8/EAAT6, SLC1A9/EAAT7), that were lost in the lineage leading to human and mouse. Interestingly members of these two subfamilies can also be found in prototherian, sauropsidan, and amphibian genomes, suggesting that these genes are specifically retained in these lineages.

\section{Results}

The zebrafish genome contains 13 slc1 genes on separate chromosomal locations

As a basis to study the evolutionary history of a given gene family, we analyzed the abundance of $s l c 1$ genes in the zebrafish genome. While in the human and mouse 7 different members of the $s l c 1$ gene family have been described, we identified and annotated 13 slc1 family genes in the zebrafish genome. Cloning from whole embryo cDNA indicated that all of the 13 annotated slc1 sequences are indeed transcribed. Sequencing of the amplified cDNA fragments revealed no significant deviation from our predicted sequences, except for some sites of single nucleotide polymorphisms.

The fact that the slc1 gene family in zebrafish consists of nearly twice as many genes as present in genomes of human and mouse suggests that at least some of the zebrafish $s l c 1$ orthologs have originated from the teleost specific whole-genome duplication. Physical and virtual mapping indicated that none of the sequences within a subgroup of $s l c 1$ are located within the same chromosomal cluster (Additional File 1), supporting the hypothesis that the genes originated from whole genome duplications rather than from individual tandem duplications.

Gene duplicates originating from whole genome duplications should be parts of large blocks of duplicated gene pairs, called paralogons. This holds true for slc1a3a/ eaat $1 a$ and slc1a3b/eaat1b, which are located on chromosome 10 and 5 respectively. Moreover, these chromosomes are derived from a common protochromosome, 
further supporting a whole genome duplication event $[7,8]$.

The microsynteny analysis of slc1a2a/eaat $2 a$ and slc1a2b/eaat $2 b$ revealed that these genes are located within two chromosomal regions on chromosomes 7 and 25 that show multiple duplicated genes (Fig. 1a). These results confirm the assumption that also the chromosomes 7 and 25 are derived from a common protochromosom \{Taylor, 2003 \#97; Postlethwait, 2000 \#208\} The microsynteny analysis for the slc1a7a/eaat5a and slc1a7b/eaat $5 b$ genes however gave a more complex pattern. While the slc1a7b/eaat $5 b$ gene is located on a small chromosomal fragment on chromosome 23 that shows only a handful of genes with conserved synteny to the human chromosome 1 , the slc1a7a/eaat5a gene is located on chromosome 2 harbouring many genes that have a similar alignment on the human chromosome 1 (Fig. 1b). Strikingly the SCP2 gene, which on the human chromosome is adjacent to the SLC1A7/EAAT5 gene can also be found next to slc1a7a/eaat $5 a$ and slc1a $7 b / e a a t 5 b$ genes in zebrafish, indicating that this region has been as expected duplicated during evolution.

\section{Evidence for the existence of additional SLC1 subgroups}

We subsequently analyzed the phylogenetic relationships of the identified zebrafish slc1 genes with their corresponding human and mouse orthologs. Interestingly, zebrafish slc1 family members do not as expected segregate into 7 clearly distinguishable subgroups with two orthologs each, but rather show a variable number of zebrafish paralogs within the different groups (see Fig. 2 and Additional File 2). While in zebrafish only one ortholog for SLC1A1/EAAT3, SLC1A4, SLC1A5 and SLC1A6/EAAT4 exists, two orthologs for SLC1A3/ EAAT1, three orthologs for SLC1A2/EAAT2 and even four genes most closely related to SLC1A7/EAAT5 were found, suggesting that in zebrafish additional slc1 subgroups exist.

Given this unexpected result, we analyzed slc1 gene families in other teleost genomes (Oryzias latipes, Takifugu rubripes, Gasterosteus aculeatus). Even teleost genomes only distantly related to zebrafish displayed an almost identical number and phylogeny of slc1 family members as those identified in the zebrafish genome (Fig. 3 ), suggesting that within the teleost lineage this number of slc1 family members is common. Moreover, members within the defined subgroups slc1a2/eaat2 and slc1a7/ eaat 5 clearly segregate into two separate branches, suggesting that in teleosts two additional slc1 subgroups, now named slcla 8 and slc1a9, exist.

This assumption was supported by an analysis of the intron/exon structure of the additional subfamilies. While we found a high conservation of the exon number and their corresponding lengths within a given subgroup, the exon size in the newly defined subfamilies were altered (Fig. 4).

\section{SLC1 gene retention and loss across vertebrate lineages}

Since we saw the loss of two SLC1 subfamilies while comparing teleosts and euarchontoglires (rodents and primates), we decided to extend our analysis to encompass the whole vertebrate tree (Fig. 5A; for a complete list of species analyzed see Additional File 3). Therefore we analyzed the genomic information from species, covering the main vertebrate lineages including marsupials, monotremata, sauropsides, and amphibiens (Fig. 5A). While the genomic information from the species used for the following analysis cover the main vertebrate lineages, a complete set of data can be found in Additional File 3 (common and scientific names, abbreviations and genomic coverage), Additional File 4 (links to genomic and transcript information), and Additional File 5 (intron/exon sizes of all species analyzed).

Database analyses indicated that all therian species have lost SLC1A8 and SLC1A9. In contrast, the members of egg laying vertebrates (prototheria, amphibia, and sauropsida) display various gene losses and retentions (Fig. $5 \mathrm{~B})$. Monotremates (prototheria) and birds have retained SLC1A8 and lost SLC1A9, whereas the reverse pattern holds true for lepidosauromorphes (lizards) that have retained SLC1A9 and lost SLC1A8. Xenopus tropicalis, as a representative of the amphibians on the other hand has retained members of both subfamilies. Interestingly, additional gene losses occurred in a variety of lineages. For instance, chicken (Gallus gallus) has lost SLC1A5 and the green anole lizard (Anolis carolinensis) has lost SLC1A6 (Fig. 5B).

Phylogenetic reconstruction including now SLC1 sequences from teleosts, amphibia, sauropsida and mammalia clearly indicates that the newly assigned SLC1A8 and SLC1A9 each form a separate clade (Fig. 5C).

In order to get an additional glimpse at the evolutionary history of SLC1 genes, we analyzed the genomes of two basal vertebrates, namely the elephant shark (Callorhinchus milii) and the sea lamprey (Petromyzon mari$n u s)$. The elephant shark, belonging to the cartilaginous fish, represents an independent evolutionary branch of the vertebrates, whose genomes are thought to have undergone the same number of genome duplications as the mammalian branch [25]. The low $(1.4 \times)$ coverage of the elephant shark genome prevented the assembly of complete slc1 cDNA sequences. Nevertheless, our comparison revealed only exons for one ortholog per subfamily, with the notable exception of the SLC1A2/A9 subgroup, where we detected a retained SLC1A9 ortholog. Interestingly we also identified two paralogs of the SLC1A2/A9 subgroup in the lamprey, a jawless verte- 
brate at the base of the vertebrate tree (for a phylogenetic tree see Additional File 6).

\section{Chromosomal location of putative human SLC1A8 and SLC1A9 remnants}

In order to see if remnants of the SLC1A8 and SLC1A9 genes can still be found within the human genome, we performed a microsynteny analysis localizing genes flanking SLC1A8 and SLC1A9. Interestingly, zebrafish slc1a8a and slc1a8b are both flanked by elavl1, a gene that can be located to human chromosome 19 (Fig. 6). This human genomic region shows a highly conserved synteny with regions flanking the zebrafish slcla8 genes, suggesting that the inactivated human SLC1A8 gene might originally have been located on chromosome 19 in the p13.2/3 region (Fig. 6). Interestingly, the putative inactivated human ortholog of slc1a9 has also been located on chromosome 19, however in contrast to SLC1A8, which is located on the shorter arm of the chromosome, SLC1A9 has been located on the longer arm in the region q13.3. This region also displays a highly conserved synteny, implying that the inactivated gene might indeed have been part of this chromosomal region. However, despite extensive attempts we were not able to identify sequences of the inactivated human SLC1A8 and SLC1A9 genes, suggesting that the degeneration of these genes has already progressed too far to allow detection.

Furthermore synteny analysis in Xenopus and chicken also revealed ELAVL1 in close proximity to SLC1A8 within the same chromosomal fragment. Similarly we found the NUCB1 gene in the vicinity of SLC1A9 in the Xenopus and Anolis genome (data not shown).

\section{The expression patterns of zebrafish slc1a3/eaat 1 and slc1a2/eaat2 paralogs suggest subfunctionalization}

As an example to compare the spatial and temporal expression pattern of duplicated genes, we investigated

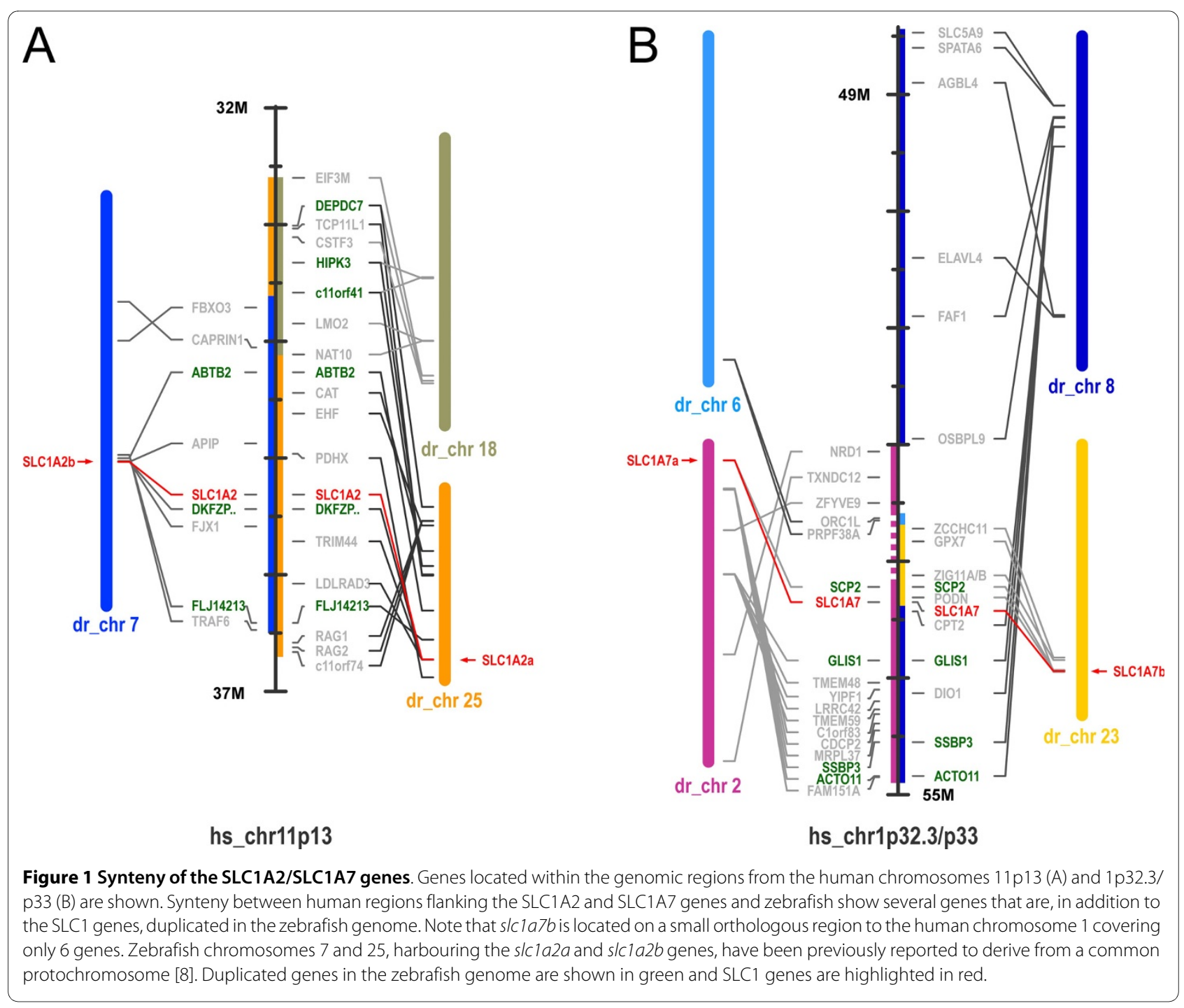




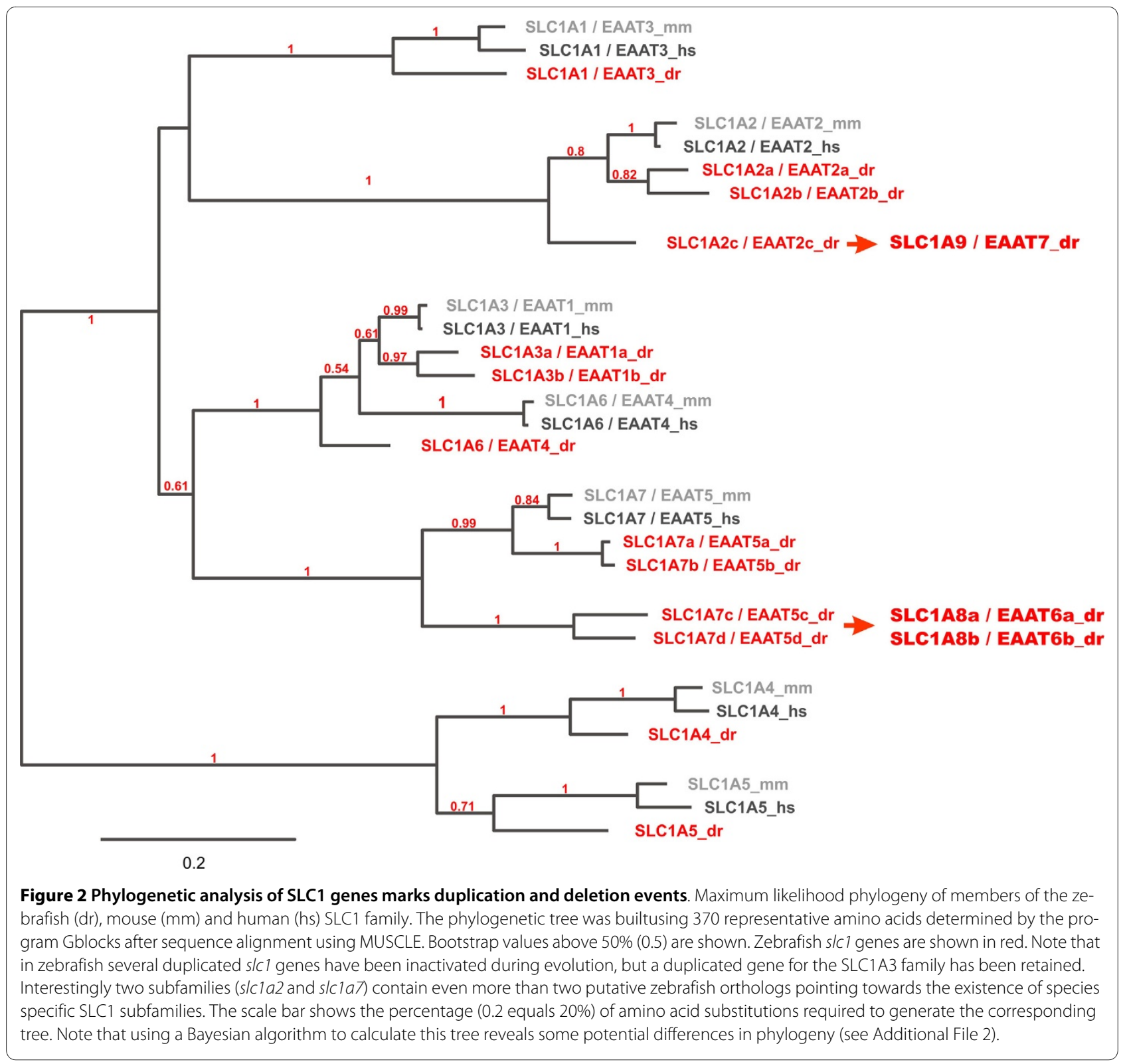

the expression patterns of the zebrafish slc1a3 and slc1a2 subfamily genes.

Whole-mount in situ hybridization experiments show that slc1a3a transcripts can be found in glia cells of the larval zebrafish brain at 3 days post fertilization (Fig. 7A and $7 \mathrm{~B})$, similar to the expression of mammalian SLC1A3/EAAT1 in astrocytes of the brain.

We found non-overlapping expression for the slc1a3b/ eaat $1 b$ paralog in neuromasts of the lateral line organ (Fig. 7C and 7D). Neuromasts consist of sensory hair cells and support cells that are homologous to mammalian inner ear hair cells and their adjacent support cells. The observed expression is consistent with a comparable function of this transporter in neuromasts, as observed for the ribbon synapse of the mammalian inner ear [26].
The slc1a2/eaat2 paralogs are both expressed in glia cells of the larval zebrafish brain (Fig. 7E-H) in a similar pattern to slc1a3a/eaat1a, which is consistent with SLC1A2/EAAT2 expression in mammalian astrocytes. In the retina, however, we found a complementary expression pattern with slc1a2a/eaat2a transcripts in the inner (Fig. 7E and 7F, arrow) and slc1a2b/eaat $2 b$ transcripts in the outer retina (Fig. 7H, arrow).

While the expression of zebrafish slc1a2b/eaat $2 b$ paralogs is in line with mammalian slc1a2/eaat2 expression in the outer retina, the slc1a2a/eaat $2 a$ expression in the inner retina has not been reported in mammals, suggestive of neofunctionalization.

Taken together our expression data for the slc1a3/eaat1 and slc1a2/eaat2 paralogs reveal subfunctionalization 


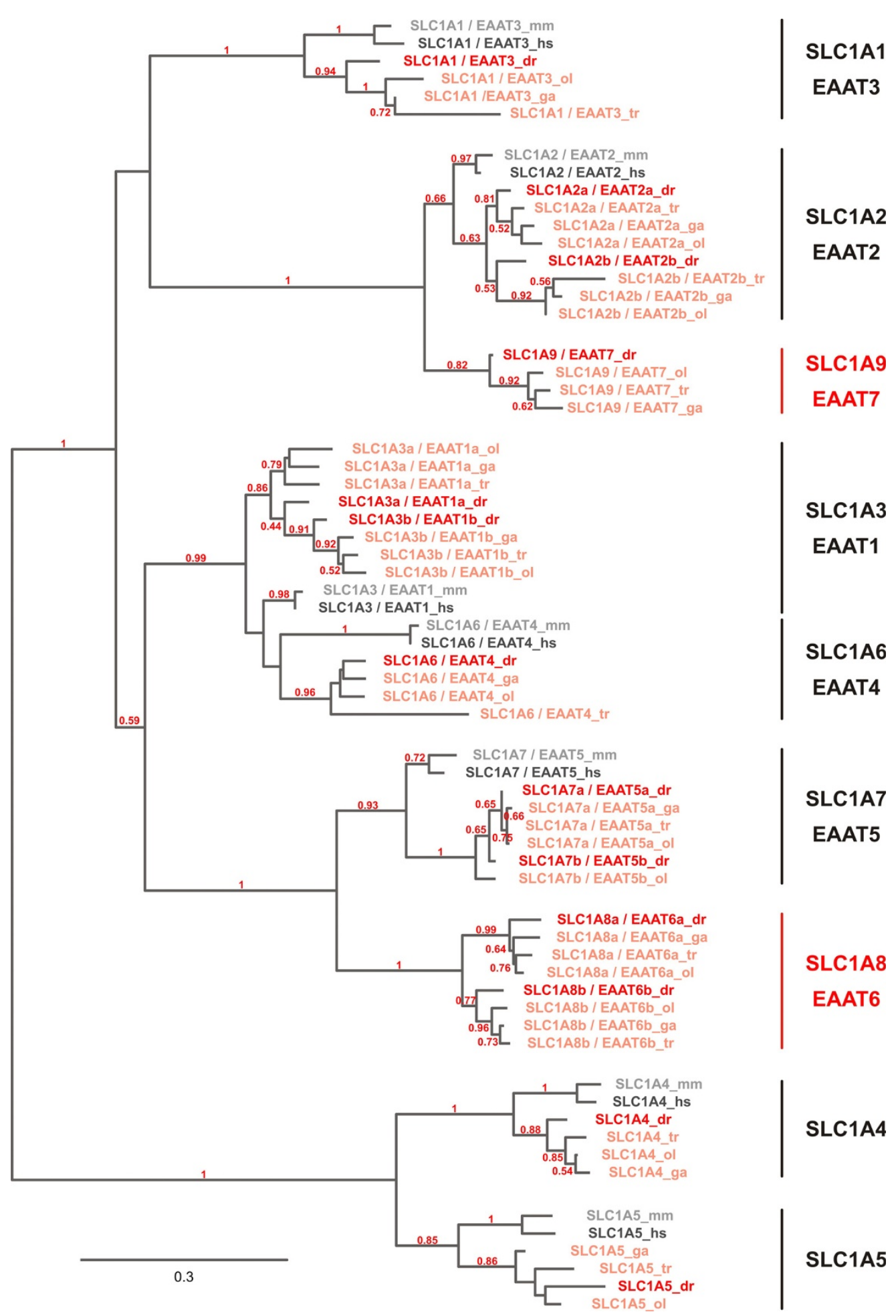

Figure 3 Teleosts display a conserved number of retained slc1 genes. For this analysis the following species in addition to mouse ( $\mathrm{mm}$ ) and human (hs) were used: Zebrafish Danio rerio (dr), Takifugu rubripes (tr), Medaka Oryzias latipes (ol) and the stickleback Gasterosteus aculeatus (ga). The phylogenetic tree was build using the maximum likelihood method on a 331 aa stretch determined by the program Gblocks after MUSCLE alignment of the full-length SLC1 amino acid sequence. Bootstrap values above 50\% (0.5) are shown. While zebrafish s/c1 genes are shown in dark red, other teleost s/c1 genes are highlighted in light red. Within the teleost family the number and phylogeny of s/cl genes is highly preserved, with the exception of s/c1a7b/eaat5b which could neither be found in stickleback nor in torafugu. In case of s/cla9 the takifugu rubripes gene was replaced by the Tetraodon gene as the takifugu s/c1a9gene could only be partly assembled. Note that teleost $\mathrm{s} / \mathrm{c} / \mathrm{a} 2 \mathrm{c} / \mathrm{eaat} 2 \mathrm{c}$ and $\mathrm{s} / \mathrm{c} / \mathrm{a} / \mathrm{c} / \mathrm{d} / \mathrm{eaat} 5 \mathrm{c} / \mathrm{d}$ genes are phylogenetically clearly separated from other SLC1 subfamilies pointing towards the existence of two additional species specific subfamilies now called s/cla8 and slc1a9. The scale bar shows the percentage of amino acid substitutions required to generate the corresponding tree. 


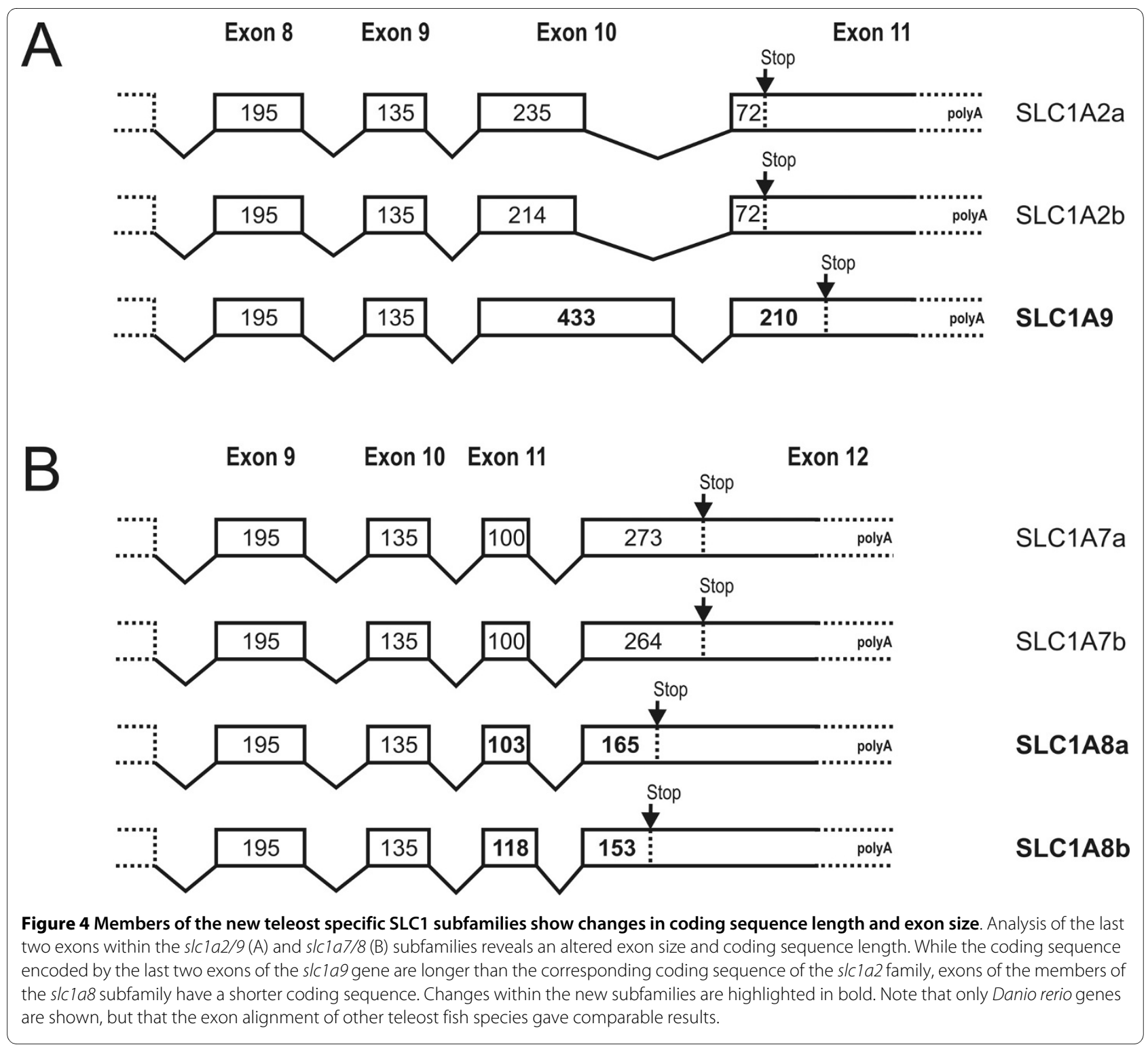

events in the zebrafish, since each paralog fulfills presumably parts of the ancestral function as predicted by the Duplication Degeneration-Complementation model $[11,12]$.

\section{Discussion}

Modern genomes are shaped by frequent duplication and deletion events. Most dramatic are whole genome duplications that were prominently stated by Susumo Ohno as the most important driving force in the evolution of metazoans [1]. This idea has immediate appeal since it provides an elegant route to the generation of new functions without compromising essential ancestral gene functions. Following the split from invertebrates, modern vertebrate genomes harbour remnants of two whole genome duplications $[2,3]$, and there is now overwhelm- ing evidence that ray-finned fishes (teleosts) had an additional whole genome duplication dating back about 320350 million years ago (Mya) [5], leading to many unique teleost specific genes. While analyzing solute carrier family 1 (slc1) genes in zebrafish, we found as expected evidence for many duplicated genes. However, some zebrafish slc1 genes turned out to be not the result of the teleost specific R3. Further analyses ruled out the possibility that these SLC1 genes originated from the teleost specific genome duplication. One possible explanation for these additional genes is lineage and species specific gene loss. This has long been recognized in unicellular organisms $[27,28]$. However more recent reports demonstrate that such events are also observed in vertebrate species [16,29-31], where the impact of such species specific gene loss remains unclear. In the case of the SLC1 
A

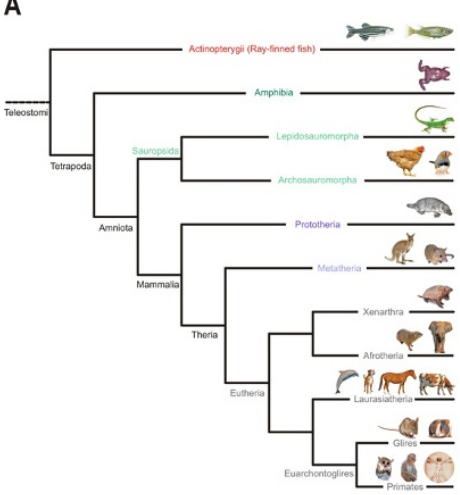

B

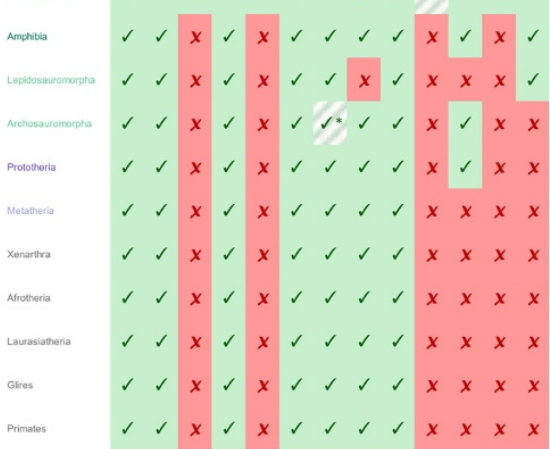

C

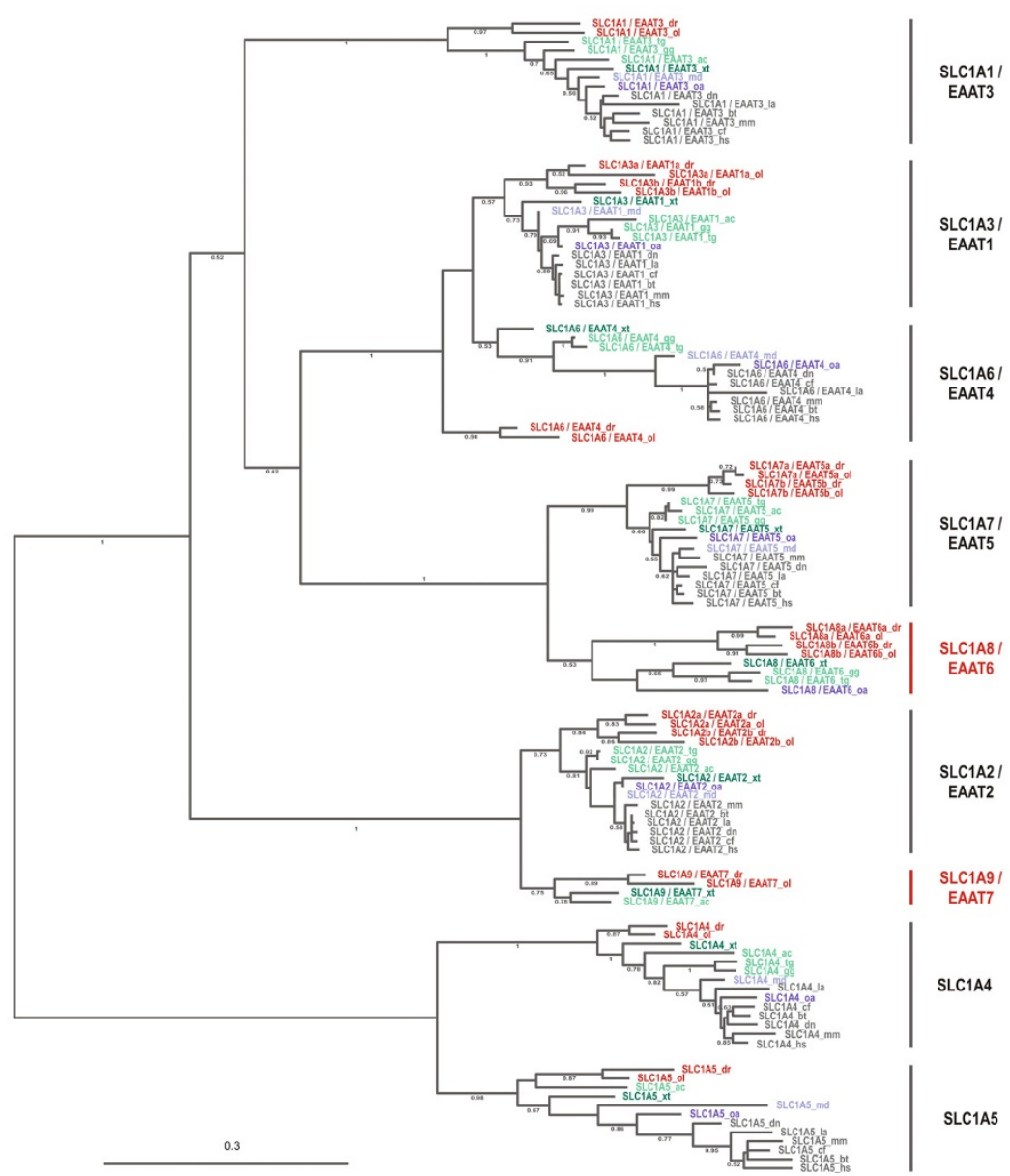

Figure 5 Selective gene loss within the vertebrate lineage leads to species specific SLC1 subfamilies. (A) Schematic representation of the vertebrate phylogenetic tree. Major superclasses, classes and infraclasses are indicated and color coded for easier comparison and identification. The species used in the following phylogenetic analysis are shown by representative pictures. (B) The number of active SLC1 genes varies within different species. Due to the fish specific genome duplication (R3), ray finned fish have an increased number of SLC1 genes as indicated by the presence of 'b' paralogs, which cannot be found in other vertebrates. Note that also within the actinopterygian lineage selective gene loss occurs (striped background) as no SLC1 A7b paralog can be found in the medaka genome. Within the tetrapod lineage, theria have lost both the SLC1 A8 and the SLC1 A9 gene, whereas prototheria and archosauromorpha have only lost the SLC1A9 and lepidosauromorpha have lost the SLC1A8 gene. Further gene loss can be observed in some avian species (SLC1A5) and in the green lizard Anolis carolinensis (SLC1A6). (C) Maximum likelihood phylogeny of analyzed vertebrate species. The phylogenetic tree was build using 361 representative amino acids residues determined by the program Gblocks after sequence alignment using MUSCLE. Bootstrap values above 50\% (0.5) are shown. Species belonging to the different superclasses, classes and infraclasses are highlighted in the same colors as used in Figure 5A. Note that members of the newly identified SLC1A8 and SLC1 A9 subgroups all group together forming indeed a separate branch within the SLC1 tree (highlighted in red). The scale bar shows the percentage (0.3 equals $30 \%)$ of amino acid substitutions required to generate the corresponding tree. 
family, where five of the seven human and mouse SLC1 genes are grouped in the functionally defined excitatory amino acid transporter (EAAT) family, in teleosts we found members of two additional subfamilies, now called SLC1A8/EAAT6 and SLC1A9/EAAT7, in teleosts. These two subfamilies have been lost in all therian mammals. This is quite surprising, as these proteins are involved in the removal of glutamate from vertebrate synapses and are therefore likely under immediate selective pressure.

In order to reconstruct the phylogenetic history of this gene family we studied a number of vertebrate species from all major vertebrate lineages. We found representatives of both new subfamilies in all analyzed teleost species, showing that the existence of these genes is common to teleost species and is not restricted to zebrafish. Analysis of amphibian genomes reveal the existence of one member of each SLC1A8/EAAT6 and SLC1A9/EAAT7 subfamily, arguing that the tetrapod ancestral species still contained the full complement of SLC1 subfamilies. In this respect it is interesting to note that a study of glutamate transporters in the tiger salamander Ambystoma tigrinum retina reports the cloning of a SLC1A8/EAAT6, as well as a SLC1A9/EAAT7 gene (referred to as EAAT2b and EAAT5b in the report, respectively) [32,33], which indeed group into the two new subfamilies (data not shown). These findings provide additional evidence for a scenario where the genes of these subfamilies have been lost after the amphibia - amnionta split.

A clue to when the first subfamily was lost is provided by the sauropsida. Here the Lepidsauromorpha (represented by the green anole lizard Anolis carolinensis) have retained SLC1A9 but lost SLC1A8. The situation is reversed in the archosauromorpha (represented by the bird species zebrafinch and chicken), where SLC1A9 was lost but SLC1A8 was retained. This suggests that the genome of the last common ancestor of the sauropsida still contained both subfamilies, followed by independent gene loss in the two branches of the sauropsida. Following this logic the genome of the amniote ancestors should have contained members of both subfamilies.

In mammals only the protherian species (represented by the duck-billed Platypus Ornithorhynchus anatinus) have retained the member of SLC1A8, while all other remaining mammals have lost members of both subfamilies. In the most parsimonious scenario the last common mammalian ancestor has already lost SLC1A9 but has retained SLC1A8. Subsequently SLC1A8 was lost at the base of the therian lineage, whereas a member of this subfamily was retained in the branched off protheria species. Hence the SLC1A8 and SLC1A9 subfamilies have been lost independently in a number of lineages. While for instance SLC1A8 has been lost at least twice independently in the archosauromorpha and therian lineage, SLC1A9 has been lost independently in the therian and lepidosauromorpha lineage. In order to infer the ancestral situation we assessed the SLC1/EAAT repository in species that are at the base of the gnathosomes.

Genome information for two species of interest, the elephant shark (Callorhinchus milii) and the sea lamprey (Petromyzon marinus), is available. While the former represents the cartilaginous fish, an independent branch of the vertebrates that has split before the division of the tetrapod and the teleost lineage, the later belongs to a clade of skulled chordate animals lacking jaws. Since both lineages branched off before R3, cartilaginous fish as well as lampreys are expected to have undergone the same number of genome duplications as the mammalian branch. Consequently the gene repertoire between lamprey, sharks, and mammals should be comparable [25,34]. While we found for each therian SLC1 gene a corresponding elephant shark ortholog, we identified several exons of a retained SLC1A9 ortholog, demonstrating that this basal species have retained members of additional SLC1 gene families. Interestingly we found only one exon sequence for a putative SLC1A8/EAAT6 ortholog in the lamprey and no trace of this gene in the elephant shark. This suggests that either this gene has been lost independently in both of these basal species (assuming that the putative lamprey exon is a false positive one) or that limited genomic information prevented us from identifying these corresponding genes.

Up to now, we have focused our discussion mainly on the SLC1A8 and SLC1A9 subfamilies. An interesting case is also the loss of SLC1A6/EAAT4 in the green anole lizard. This gene is not duplicated in any species analyzed and is prominently expressed in the cerebellum. We can only speculate that another SLC1 gene in lizards must have taken over the function of SLC1A6 in glutamate removal from cerebellar synapses. Support for such a scenario comes from a study comparing the similarity of the glutamatergic sytem in the turtle Chrysemys picta and rodents [35].

We have documented multiple gene loss in vertebrate lineages, raising the question if traces of these lost genes can still be found in extant genomes. Most of these gene losses must have happened many Mya ago, so finding defunct gene sequences is rather unlikely. However by microsynteny analyses, we found the putative genomic location of both lost SLC1 genes in the human genome. Intriguingly both lost genes were most likely located on human chromosome 19. While the putative SLC1A8 location is on the short arm of chromosome 19 (19p13.2/ 3 ), the most likely position of the inactivated SLC1A9 is on the long arm at position $19 \mathrm{q} 13.3$. For this human chromosome extensive rearrangements within the short arm and many intrachromosomal breaks in the long arm have been reported, even since the time of primate rodent divergence [36]. Therefore it seems well conceivable that 

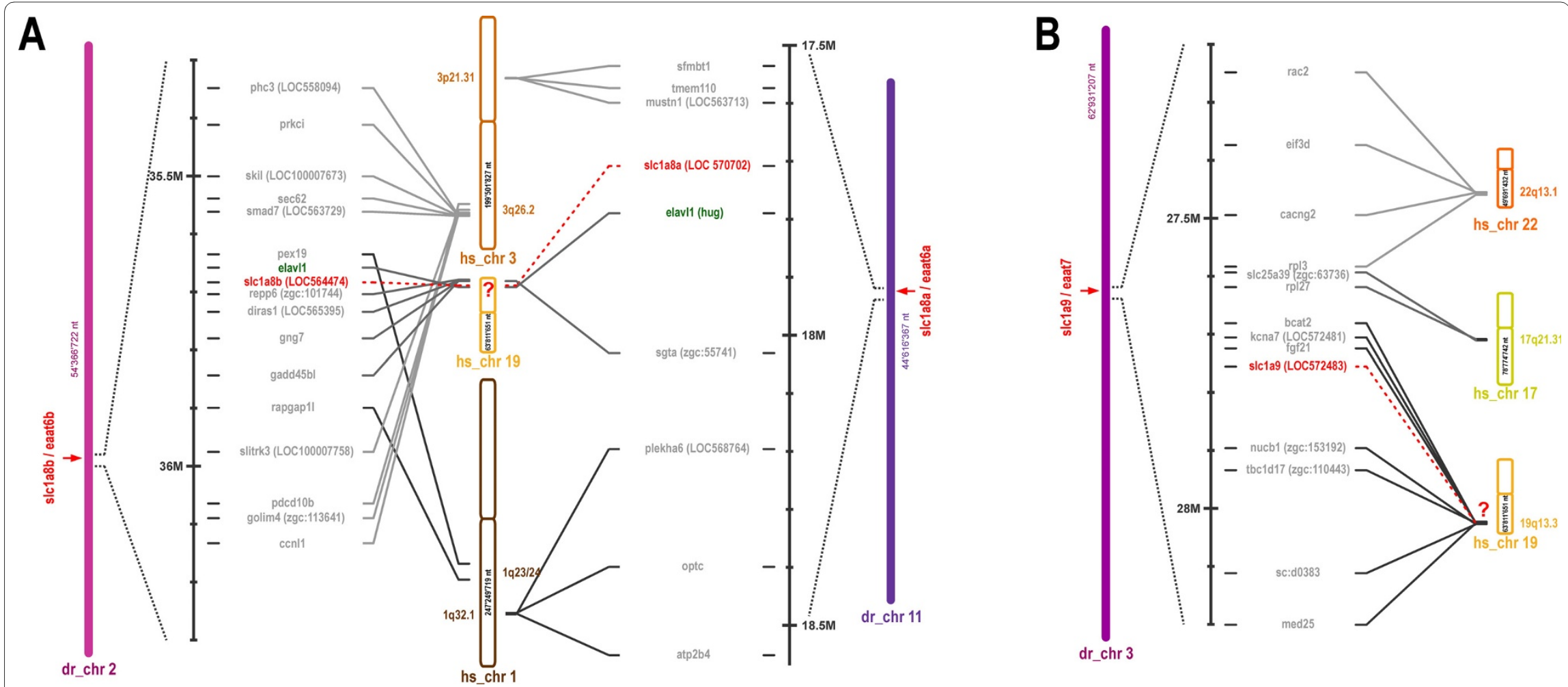

Figure 6 Synteny of the s/c1a8/s/c1a9 gene family. Genomic regions around the new zebrafish s/cla8/slc1a9 subfamilies are shown. SLC1 genes are highlighted in red. (A) Adjacent to both, the s/c1a8a and the s/c1a8b gene respectively, the elavl1 gene (highlighted in green) can be found. In addition multiple genes flanking the s/cla8 loci localize to the same human chromosomal region (19p13.2/3), (B) Zebrafish genes adjacent to slcla9 can be found on the long arm of the human chromosome 19 in the region q13.3. Note that several genes upstream and downstream of the s/c $199 \mathrm{genes}$ are localized within a narrow band (1,6 $\times 10^{6}$ bases) on chromosome 19 . 


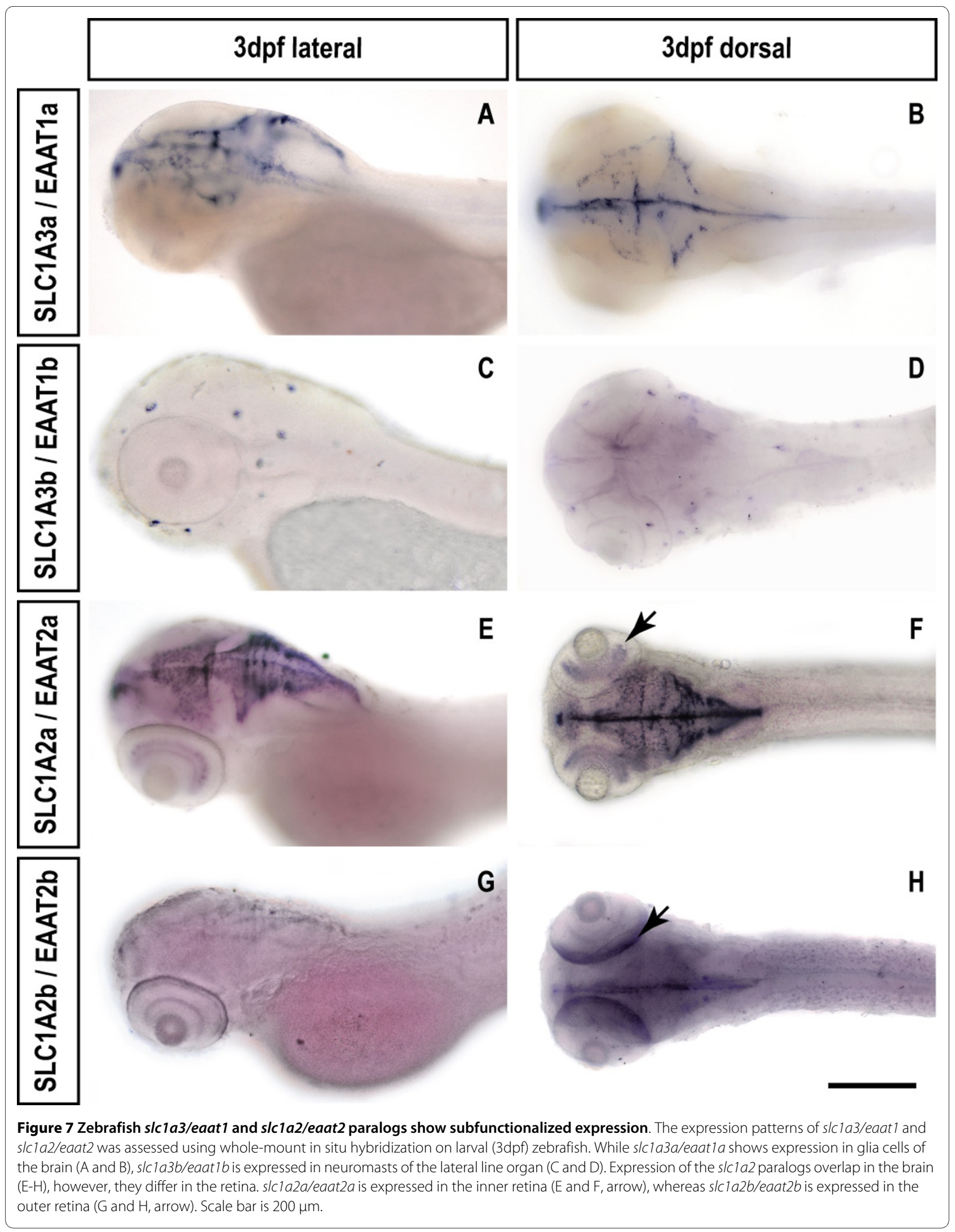


many genes, including SLC1A8 and SLC1A9, may have been rendered non-functional during these rearrangement events. Our microsynteny analyses indicated that the ELAVL1 gene is in close proximity of both zebrafish SLC1A8 genes, and that other genes in this region map like ELAVL1 to the human chromosome 19. Interestingly this synteny is conserved in chicken and Xenopus, where ELAVL1 and SLC1A8 are neighbors on chromosome 28 (890K-930K) and scaffold 112 (300K-400K) respectively (data not shown).

\section{Conclusion}

The phylogenetic analysis of a gene family spanning multiple taxa can yield important information about their evolutionary history. In the case of the solute carrier family 1 , consistent with the duplication-complementationdegeneration model, most of the 7 human or mouse orthologs have one or two teleost orthologs. However, for two teleost SLC1 subfamilies we found no corresponding therian counterpart. This indicates that therians must have lost these two subfamilies, now named SLC1A8/ EAAT6 and SLC1A9/EAAT7.

These two subfamilies are not teleost specific, but can be found throughout different vertebrates. An analysis of the major vertebrate lineages revealed an intriguing pattern of lineage specific gene losses, shaping the phylogenetic history of SLC1 genes. In all non-therian vertebrate species we found at least one member of the SLC1A8/9 subfamilies. Amphibians have neither lost SLC1A8 nor SLC1A9, lepidosauria (extent lizards, snakes and tuatara) on the one hand have lost the SLC1A8 ortholog, while birds on the other hand have lost SLC1A9. Interestingly egg laying mammals (platypus) are the only mammals that have also retained a SLC1A8 ortholog.

By studying one gene family across a number of vertebrate taxa, inferences can be made about the evolutionary history of these genes. Such an analysis is needed to guide the interpretation of functional data obtained for those genes and provides a fascinating scenario to study the evolution of vertebrate gene families.

\section{Methods}

\section{Zebrafish maintenance and breeding}

Wild-type fish of the Tübingen (Tü) inbred strain were bred and crossed as previously described [37]. Embryos were raised in $\mathrm{E} 3$ medium $(5 \mathrm{mM} \mathrm{NaCl}, 0.17 \mathrm{mM} \mathrm{KCl}$, $0.33 \mathrm{mM} \mathrm{CaCl}_{2}, 0.33 \mathrm{mM} \mathrm{MgSO}_{4}$ ) at $28^{\circ} \mathrm{C}$. Stages refer to the development in days post fertilization (dpf).

\section{Annotation of zebrafish SLC1 cDNAs}

As many genes predicted within GenBank are produced by automated processes and have been shown to contain numerous errors such as missing exons and wrong predictions of start and termination sites, all cDNA sequences for the described $s l c 1$ genes were annotated manually. Sequences from the species listed in Additional File 3 were used for our analysis. Sequences were identified and annotated using combined information from EST, genome (GeneBank, http://www.ncbi.nlm.nih.gov; Ensembl, http://www.ensembl.org/index.html; version 50/51, 2008) and WGS http://blast.ncbi.nlm.nih.gov/ Blast.cgi?PAGE $=$ Nucleotides\&PRO-

GRAM=blastn\&BLAST SPEC=TraceArchive\&BLAST PROGRAMS=megaBlast\&PAGE $\_$TYPE=BlastSearch

databases. Human and mouse sequences were used as initial query. Genomic regions coding for SLC1 family genes were identified by using the tblastx alignment algorithm (for details on intron/exon sizes see Additional File 1). Corresponding genomic fragments were analyzed using the GENSCAN gene prediction program http:// genes.mit.edu/GENSCAN.html. The obtained putative cDNA and protein sequences were compared to the corresponding human and mouse orthologs and GENSCAN prediction errors were corrected by manual inspection of the intron/exon boundaries in false predicted regions (small exons and minor class splice sites are usually not recognized [38]). Gaps in the assembled sequences due to inaccurate or incomplete genome sequencing were wherever possible filled by corresponding EST or WGS sequences. Sequences found by EST and WGS searches were again blasted against the $\mathrm{nr}$ database and only if the top hits corresponded to the original query sequences were used in the annotation process. Note that in the case of duplicated genes annotation errors using WGS sequences could not be excluded due to the fact that individual exons could not be linked together. Sequence alignment of cDNA fragments and overlapping EST sequences was done using the SeqMan software (Lasergene, DNASTAR, Madison WI).

\section{Phylogenetic tree analysis}

Coding sequences of $s l c 1$ genes (except for the exon 234 analysis which was done on nucleotide sequences) were translated into proteins using the EditSeq software (Lasergene, DNASTAR, Madison WI) and obtained protein sequences were used to generate a combined sequence file in FASTA format (see Additional File 7 (protein FASTA) and Additional File 8 (exon 234 FASTA). Sequence alignment and phylogenetic analysis was performed on the Phylogeny.fr platform http://www.phylogeny.fr/version2_cgi/phylogeny.cgi. Sequences were aligned using MUSCLE (v3.7, Edgar 2004) configured for highest accuracy (MUSCLE with default settings). After alignment, ambiguous regions (i.e. containing gaps and/ or are poorly aligned) were removed with Gblocks (v0.91b, [39]) using the following parameters: minimum length of a block after gap cleaning = 10; no gap positions were allowed in the final alignment; all segments with 
contiguous nonconserved positions bigger than 8 were rejected; minimum number of sequences for a flank position $=85 \%$. The phylogenetic tree was reconstructed using the maximum likelihood method implemented in the PhyML program (v3.0 aLRT, [40]. The default substitution model was selected assuming an estimated proportion of invariant sites and 4 gamma-distributed rate categories to account for rate heterogeneity across sites. The gamma shape parameter was estimated directly from the data. Reliability for internal branch was assessed using the bootstrapping method (100 bootstrap replicates) and bootstrap values above 50\% (0.5) are shown. Graphical representation and editing of the phylogenetic tree was done using TreeDyn (v198.3, [41] and the obtained svg files were colored using the CorelDraw program. Animal icons in Figure 5 were purchased from iStockphoto http://www.istockphoto.com.

Alternative alignments and trees were calculated using the alignment programs ClustalW [42] and T-Coffee [43], and the neighbour joining (PHYLIP package 3.66 [44]; distances calculated using ProtDist [45] or MRBAYES (v3.1.2, [46] phylogeny programs. Using these alternative methods revealed some potential differences in phylogeny and a Bayesian calculated tree including human, mouse and zebrafish SLC1 sequences is shown in Additional File 2.

\section{Cloning}

Approximately $505 \mathrm{dpf}$ zebrafish larvae were homogenized to prepare total RNA using the Qiagen RNeasy kit (Qiagen), according to the manufacturer's instructions. Oligo dT primers were used to reverse-transcribe the total RNA with reverse transcriptase (First Strand Kit, Invitrogen, Carlsbad, CA). Polymerase chain reaction (PCR) was performed using sequence-specific oligonucleotide primers. Amplified fragments were ligated into the pCR-II vector (TA Cloning Kit Dual Promoter, Invitrogen, Carlsbad, CA, USA) and at least three independently amplified cDNA fragments per gene were sequenced to confirm our previously annotated zebrafish sequences. Accession numbers for the zebrafish SLC1/ EAAT genes are: SLC1A1/EAAT3 (HM138690), SLC1A2a/EAAT2a (HM138691), SLC1A2b/EAAT2b (HM138692), SLC1A3a/EAAT1a (HM138693), SLC1A3b/EAAT1b (HM138694), SLC1A4 (HM138695), SLC1A5 (HM138696), SLC1A6/EAAT4 (HM138697), SLC1A7a/EAAT5a (HM138698), SLC1A7b/EAAT5b (HM138699), SLC1A8a/EAAT6a (HM138700), SLC1A8b/EAAT6b (HM138701), SLC1A9/EAAT7 (HM138702).

\section{Genomic mapping}

All zebrafish slc1 genes except slc1a6/eaat4 were localized in the zebrafish genome by radiation hybrid mapping [47] using the LN54 panel (Loeb/NIH/5000/4000, M. Ekker, Ottawa, Canada) and specific primer pairs for the genomic sequences (see Additional File 9).

\section{Synteny analysis}

For microsynteny analyses, genes flanking the slc1 locus of human or zebrafish were used as initial queries for a tblastx [48] search against the zebrafish or human database (ncbi nr/nt database) limited to the desired species (homo sapiens, danio rerio). The different hits were compared with each other and the hit with the highest conservation (identical aa and conserved aa) was selected (note that due to frequently occurring alignment gaps, hits with the lowest expect scores (E values) not necessarily representing the ones with highest identity). Only hits displaying a significant homology in length as well as conservation were used for further analyses. The hit with the highest conservation was used in a reciprocal tblastx search against the corresponding database and only genes which identified the initially used query are depicted.

\section{Whole-mount in situ bybridization}

In vitro transcription of DIG-labeled probes was performed using the Roche RNA Labeling Kit (Roche Diagnostics, Rotkreuz, Switzerland). RNA probes were hydrolyzed to obtain fragments of about 300 bp length. Whole-mount in situ hybridization on $3 \mathrm{dpf}$ zebrafish larvae was performed as follows. PTU treated larvae were anesthetized on ice and immediately fixed in $4 \%$ paraformaldehyde in $0.2 \mathrm{M}$ phosphate buffer ( $\mathrm{pH} 7.4$ ) for 40 min at ambient temperature (RT). The in situ hybridization was performed according to the Zebrafish Book protocol [50] using an automated in situ hybridization machine (BioLane HTI, Hölle \& Hüttner, Tübingen, Germany).

\section{Additional material}

\footnotetext{
Additional file 1 Genomic localizations of s/c 1 genes in the zebrafish genome. Chromosomal localizations were identified both physically, using radiation hybrid mapping, and in silico. All zebrafish s/c1 genes localize to different chromosomal regions. For details on radiation hybrid mapping, see Materials and Methods. *The localization of s/cla6/eaat4 could not unambiguously be determined by radiation hybrid mapping and has been mapped in silico only.

Additional file 2 Phylogenetic analysis of SLC1 genes using a Bayesian algorithm. Bayesian phylogeny of members of the zebrafish (dr), mouse (mm) and human (hs) SLC1 family. The phylogenetic tree was build using 370 representative amino acids determined by the program Gblocks after sequence alignment using MUSCLE. The tree was reconstructed using the bayesian inference method implemented in the MrBayes program (v3.1.2). The number of substitution types was fixed to 6. The Poisson model was used for amino acid substitution, while rates variation across sites was fixed to "invgamma". Four Markov Chain Monte Carlo (MCMC) chains were run for 10000 generations, sampling every 10 generations, with the first 250 sampled trees discarded as "burn-in". Finally, a 50\% majority rule consensus tree was constructed. Note that in comparison to the maximum likelihood calculated tree, roots in the Bayesian tree are slightly different. While in the Bayesian build tree it appears that there is a common ancestor of SLC1A1, SLC1A2, SLC1A3 and SLC1A6, the tree deriving from maximum likelihood phylogeny suggests that there is a common ancestor between SLC1A3, SLC1A6 and SLC1A7/8 (Figure 2). Zebrafish s/c1/eaat genes are shown in red. The scale bar represents the percent of amino acid substitutions required to generate the corresponding tree.
} 


\begin{abstract}
Additional file 3 Names, abbreviations and genomic coverage of species analyzed. The common and the scientific name of the species used are indicated. The genome coverage is given. Note that coverage's highlighted in green (high coverage) and yellow (low coverage) belong to species that have been used to generate the phylogenetic tree covering the major linages. Coverage's shown in red belong to species whose sequences have been annotated but not included in the phylogenetic tree

Additional file 4 Links to transcript and genome information of the annotated sequences. The identity of the predicted and the manually annotated sequences is indicated by different colours. Identical predicted and annotated sequences are shown in green, whereas sequences with different predicted open reading frames are highlighted in red. Sequences covering the whole open reading frame are indicated as "Full" sequences and sequences lacking one or more exons are marked as partial sequences. Sequences for which neither a genomic nor a transcript link have been found are given by the letters n.d. (not detected)
\end{abstract}

Additional file $\mathbf{5}$ Intron/Exon sizes of SLC1 genes. The different SLC1 gene families are colour coded. Exons serving as identifiers for the corresponding gene family are highlighted in the identical colour as the gene family. Exons containing the start and the stop codon are highlighted in red. Intron sequences containing multiple place holders (Ns) are indicated. Additional file 6 The SLC1A9 subfamily is also present in cartilaginous fish. Database analysis on the elephant shark (Callorhinchus milii, $\mathrm{cm}$ ) and the sea lamprey (Petromyzon marinus) genome identified sequences that represent parts of the SLC1A9 gene. For the exon 234 (for more information on s/c 1 intron/exon sizes see supplementary Figure \$5), two corresponding slcla2 exons could be found, one of them representing a retained s/cla9 gene. The phylogenetic tree was build using the maximum likelihood method on the entire nucleotide sequence of corresponding 234 exons. Bootstrap values above 50\% (0.5) are shown. Elephant shark sequences are highlighted in dark red, lamprey sequences are shown in claret red and teleost genes are depicted in light red. Note that the FSGD generated two retained SLC1A2 genes and most likely also two SLC1A9 genes of which in modern teleosts only one is still present.

Additional file 7 Protein fastas of species used in the phylogenetic analysis covering the main vertebrate linages.

Additional file 8 Nucleotide fastas of the sequences used in the exon 234 analysis.

Additional file 9 Oligonucleotide Primers Used for Genomic Mapping.

\section{Authors' contributions}

MG contributed to cloning, performed the database searches and the phylogenetic analysis, annotated the s/c 1 genes, determined the intron/exon pattern

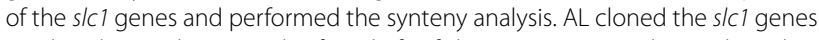
used in this study, wrote the first draft of the manuscript and contributed to expression analysis, CMM performed the expression analysis and HBS contributed to cloning and expression analysis. SCFN and MG wrote the manuscript in its present form, and conceived the project and designed experiments. Al authors have read and approved of the final manuscript.

\section{Acknowledgements}

We thank A. Bohnert for the physical mapping of the zebrafish s/c1 genes and Corinne Hodel and Dr. Edda Kastenhuber for critical reading of the manuscript. This work was supported by an ETH internal grant (TH-0-20150-3) (AL, SCFN) and by a grant of the Swiss National Science Foundation (PP00A-68868) (SCFN).

\section{Author Details}

'University of Zurich, Institute of Molecular Life Sciences, Winterthurerstrasse 190, CH-8057 Zurich, Switzerland, ${ }^{2}$ Swiss Federal Institute of Technology (ETH) Zurich, Department of Biology, Switzerland and 'Fondation BBVA-Cancer Cell Biology Programme, Spanish National Cancer Reseach Center (CNIO), E-28029 Madrid, Spain

Received: 13 January 2010 Accepted: 29 April 2010 Published: 29 April 2010

\section{References}

1. Ohno S: Evolution by Gene Duplication. Springer Verlag, New York; 1970.

2. Dehal $P$, Boore JL: Two rounds of whole genome duplication in the ancestral vertebrate. PLoS Bio/ 2005, 3(10):e314

3. Putnam NH, Butts T, Ferrier DE, Furlong RF, Hellsten U, Kawashima T, Robinson-Rechavi M, Shoguchi E, Terry A, Yu JK, et al:: The amphioxus genome and the evolution of the chordate karyotype. Nature 2008 453(7198):1064-1071.

4. Taylor JS, Braasch I, Frickey T, Meyer A, Peer Y Van de: Genome duplication, a trait shared by 22000 species of ray-finned fish. Genome Res 2003, 13(3):382-390.

5. Meyer A, Peer $Y$ Van de: From 2R to 3R: evidence for a fish-specific genome duplication (FSGD). Bioessays 2005, 27(9):937-945.

6. Jaillon O, Aury JM, Brunet F, Petit JL, Stange-Thomann N, Mauceli E, Bouneau L, Fischer C, Ozouf-Costaz C, Bernot A, et al:: Genome duplication in the teleost fish Tetraodon nigroviridis reveals the early vertebrate proto-karyotype. Nature 2004, 431(7011):946-957.

7. Kasahara M, Naruse K, Sasaki S, Nakatani Y, Qu W, Ahsan B, Yamada T, Nagayasu Y, Doi K, Kasai Y, et al:: The medaka draft genome and insights into vertebrate genome evolution. Nature 2007, 447(7145):714-719.

8. Woods IG, Wilson C, Friedlander B, Chang P, Reyes DK, Nix R, Kelly PD, Chu F, Postlethwait JH, Talbot WS: The zebrafish gene map defines ancestral vertebrate chromosomes. Genome Res 2005, 15(9):1307-1314.

9. Postlethwait JH, Woods IG, Ngo-Hazelett P, Yan YL, Kelly PD, Chu F, Huang $H$, Hill-Force A, Talbot WS: Zebrafish comparative genomics and the origins of vertebrate chromosomes. Genome Res 2000, 10(12):1890-1902

10. Brunet FG, Crollius HR, Paris M, Aury JM, Gibert P, Jaillon O, Laudet V, Robinson-Rechavi M: Gene loss and evolutionary rates following wholegenome duplication in teleost fishes. Mol Biol Evol 2006, 23(9):1808-1816.

11. Force A, Lynch M, Pickett FB, Amores A, Yan YL, Postlethwait J: Preservation of duplicate genes by complementary, degenerative mutations. Genetics 1999, 151(4):1531-1545.

12. Lynch M, Force A: The probability of duplicate gene preservation by subfunctionalization. Genetics 2000, 154(1):459-473.

13. Lynch $\mathrm{M}$, Conery JS: The evolutionary fate and consequences of duplicate genes. Science 2000, 290(5494):1151-1155.

14. Taylor JS, Peer Y Van de, Meyer A: Genome duplication, divergent resolution and speciation. Trends Genet 2001, 17(6):299-301.

15. Evans BJ: Genome evolution and speciation genetics of clawed frogs (Xenopus and Silurana). Front Biosci 2008, 13:4687-4706.

16. Feiner N, Begemann G, Renz AJ, Meyer A, Kuraku S: The origin of bmp16, a novel Bmp2/4 relative, retained in teleost fish genomes. BMCEvol Biol 2009, 9(1):277.

17. Kuraku S, Meyer A: The evolution and maintenance of Hox gene clusters in vertebrates and the teleost-specific genome duplication. Int J Dev Biol 2009, 53(5-6):765-773.

18. Amara SG, Fontana AC: Excitatory amino acid transporters: keeping up with glutamate. Neurochem Int 2002, 41(5):313-318.

19. Arriza JL, Fairman WA, Wadiche Jl, Murdoch GH, Kavanaugh MP, Amara SG: Functional comparisons of three glutamate transporter subtypes cloned from human motor cortex. J Neurosci 1994, 14(9):5559-5569.

20. Grewer C, Rauen T: Electrogenic glutamate transporters in the CNS: molecular mechanism, pre-steady-state kinetics, and their impact on synaptic signaling. J Membr Biol 2005, 203(1):1-20.

21. Huang $\mathrm{YH}$, Bergles DE: Glutamate transporters bring competition to the synapse. Curr Opin Neurobiol 2004, 14(3):346-352.

22. Kanai $Y$, Hediger MA: The glutamate/neutral amino acid transporter family SLC1: molecular, physiological and pharmacological aspects. Pflugers Arch 2004, 447(5):469-479.

23. Beart PM, O'Shea RD: Transporters for L-glutamate: an update on their molecular pharmacology and pathological involvement. $\mathrm{Br}$ J Pharmacol 2007, 150(1):5-17

24. Ryan RM, Vandenberg RJ: A channel in a transporter. Clin Exp Pharmacol Physiol 2005, 32(1-2):1-6.

25. Venkatesh B, Kirkness EF, Loh YH, Halpern AL, Lee AP, Johnson J, Dandona N, Viswanathan LD, Tay A, Venter JC, et al:: Survey sequencing and comparative analysis of the elephant shark (Callorhinchus milii) genome. PLoS Biol 2007, 5(4):e101. 
26. Glowatzki E, Cheng N, Hiel H, Yi E, Tanaka K, Ellis-Davies GC, Rothstein JD, Bergles DE: The glutamate-aspartate transporter GLAST mediates glutamate uptake at inner hair cell afferent synapses in the mammalian cochlea. J Neurosci 2006, 26(29):7659-7664.

27. Kawahara Y, Imanishi T: A genome-wide survey of changes in protein evolutionary rates across four closely related species of Saccharomyces sensu stricto group. BMC Evol Biol 2007, 7:9.

28. Aravind L, Watanabe H, Lipman DJ, Koonin EV: Lineage-specific loss and divergence of functionally linked genes in eukaryotes. Proc Natl Acad SciUSA 2000, 97(21):11319-11324.

29. Mauti O, Sadhu R, Gemayel J, Gesemann M, Stoeckli ET: Expression patterns of plexins and neuropilins are consistent with cooperative and separate functions during neural development. BMCDev Biol 2006, 6:32.

30. Tian X, Pascal G, Fouchecourt S, Pontarotti P, Monget P: Gene birth, death, and divergence: the different scenarios of reproduction-related gene evolution. Biol Reprod 2009, 80(4):616-621.

31. Demuth JP, Hahn MW: The life and death of gene families. Bioessays 2009, 31(1):29-39.

32. Eliasof S, Arriza JL, Leighton BH, Kavanaugh MP, Amara SG: Excitatory amino acid transporters of the salamander retina: identification, localization, and function. J Neurosci 1998, 18(2):698-712.

33. Elias of S, Arriza JL, Leighton BH, Amara SG, Kavanaugh MP: Localization and function of five glutamate transporters cloned from the salamander retina. Vision Res 1998, 38(10):1443-1454.

34. Kuraku S, Meyer A, Kuratani S: Timing of genome duplications relative to the origin of the vertebrates: did cyclostomes diverge before or after? Mol Biol Evol 2009, 26(1):47-59

35. Keifer J, Carr MT: Immunocytochemical localization of glutamate receptor subunits in the brain stem and cerebellum of the turtle Chrysemys picta. J Comp Neurol 2000, 427(3):455-468

36. Grimwood J, Gordon LA, Olsen A, Terry A, Schmutz J, Lamerdin J, Hellsten U, Goodstein D, Couronne O, Tran-Gyamfi M, et al:: The DNA sequence and biology of human chromosome 19. Nature 2004, 428(6982):529-535.

37. Mullins MC, Hammerschmidt M, Haffter P, Nusslein-Volhard C: Large-scale mutagenesis in the zebrafish: in search of genes controlling development in a vertebrate. Curr Biol 1994, 4(3):189-202.

38. Patel $A A$, Steitz JA: Splicing double: insights from the second spliceosome. Nat Rev Mol Cell Biol 2003, 4(12):960-970.

39. Castresana J: Selection of conserved blocks from multiple alignments for their use in phylogenetic analysis. Mol Biol Evol 2000, 17(4):540-552.

40. Guindon S, Gascuel O: A simple, fast, and accurate algorithm to estimate large phylogenies by maximum likelihood. Syst Bio/ 2003, 52(5):696-704.

41. Chevenet F, Brun C, Banuls AL, Jacq B, Christen R: TreeDyn: towards dynamic graphics and annotations for analyses of trees. $B M C$ Bioinformatics 2006, 7:439.

42. Thompson JD, Higgins DG, Gibson TJ: CLUSTAL W: improving the sensitivity of progressive multiple sequence alignment through sequence weighting, position-specific gap penalties and weight matrix choice. Nucleic Acids Res 1994, 22(22):4673-4680.

43. Notredame C, Higgins DG, Heringa J: T-Coffee: A novel method for fast and accurate multiple sequence alignment. J Mol Biol 2000, 302(1):205-217.

44. Felsenstein J: Phylogeny interface package. Cladistics 1989, 5:164-166.

45. Elias I, Lagergren J: Fast computation of distance estimators. BMC Bioinformatics 2007, 8:89.

46. Huelsenbeck JP, Ronquist F: MRBAYES: Bayesian inference of phylogenetic trees. Bioinformatics 2001, 17(8):754-755

47. Walter MA, Spillett DJ, Thomas P, Weissenbach J, Goodfellow PN: A method for constructing radiation hybrid maps of whole genomes. Nat Genet 1994, 7(1):22-28.

48. Altschul SF, Gish W, Miller W, Myers EW, Lipman DJ: Basic local alignment search tool. J Mol Biol 1990, 215(3):403-410.

49. Karlsson J, von Hofsten J, Olsson PE: Generating transparent zebrafish: a refined method to improve detection of gene expression during embryonic development. Mar Biotechnol (NY) 2001, 3(6):522-527.

50. Westerfield M: THE ZEBRAFISH BOOK: A guide for the laboratory use of zebrafish (Danio rerio). Eugene: University of Oregon Press; 2000. doi: 10.1186/1471-2148-10-117

Cite this article as: Gesemann et al., Phylogenetic analysis of the vertebrate Excitatory/Neutral Amino Acid Transporter (SLC1/EAAT) family reveals lineage specific subfamilies BMC Evolutionary Biology 2010, 10:117

\section{Submit your next manuscript to BioMed Central and take full advantage of:}

- Convenient online submission

- Thorough peer review

- No space constraints or color figure charges

- Immediate publication on acceptance

- Inclusion in PubMed, CAS, Scopus and Google Scholar

- Research which is freely available for redistribution

Submit your manuscript at www.biomedcentral.com/submit
C BioMed Central 